\title{
Adaptação transcultural do Teste de Conhecimento Emocional: avaliação neuropsicológica das emoções
}

\author{
Nara Côrtes Andrade - Universidade de São Paulo e Universidade Católica do Salvador, Salvador, Brasil \\ Neander Abreu - Universidade Federal da Babia, Salvador, Brasil \\ Igor Menezes - Universidade Federal da Babia, Salvador, Brasil \\ Clándia Berlim de Mello - Centro Paulista de Neuropsicologia, Universidade Federal de São Paulo, São Paulo, Brasil \\ Victor Riccio Duran - Universidade Federal da Babia, Salvador, Brasil \\ Narena de Alencar Moreira - Universidade Federal da Babia, Salvador, Brasil
}

\begin{abstract}
Resumo
As emoções desempenham um papel central nas relações das crianças e déficits na compreensão de emoções têm sido associados a diversos transtornos neuropsiquiátricos na infância. Entretanto, verifica-se uma escassez de instrumentos psicológicos disponíveis no Brasil que avaliem esta habilidade. O presente estudo teve por objetivo adaptar transculturalmente o Teste de Conhecimento Emocional (EMT). O instrumento original foi traduzido por uma equipe de pesquisadores bilíngues e posteriormente retraduzido para o inglês. As versões preliminares foram julgadas pelos autores do instrumento e por juízes brasileiros especialistas. A versão final foi aplicada em uma amostra de 50 crianças entre três e seis anos de idade e respondida por nove juízes de três estados brasileiros. Os resultados mostram uma boa equivalência semântica, além de bons níveis de concordância quanto às respostas $(x=0,88, Z=95,2, p<0,001)$. A versão final do EMT mostrou-se adequada e satisfatória.
\end{abstract}

Palavra-chave: Emoção; Crianças; Testes neuropsicológicos.

Transcultural adaptation of the Emotion Matching Task: an emotion neuropsychological assessment

\begin{abstract}
Emotions play a central role in children's relationships. Deficits in emotional understanding have been associated with several neuropsychiatric disorders. In Brazil, however, few psychological instruments are available to assess young children's emotional development. The objective of the present study was to make a transcultural adaptation of the Emotion Matching Task (EMT). The EMT was translated and adapted by a team of bilingual researches and then back-translated to English. The preliminary versions were assessed by EMT's authors and by Brazilians specialized judges. The final version was applied in a sample of 50 children between three and six years of age and answered by nine judges in three Brazilian states. The results indicate good semantic equivalence and good agreement with the answers provided $(x=0.88, Z=95.2, p<0.001)$. The final version of the EMT was considered appropriate and satisfactory.

Keywords: Emotion; Child; Neuropsychological Test.
\end{abstract}

Adaptación transcultural del Test de Conocimiento Emocional: evaluación neuropsicológica de las emociones

\begin{abstract}
Resumen
Las emociones desempeñan un papel central en las relaciones y déficits en la comprensión de emociones ha sido asociado a diversos trastornos neurológicos y psiquiátricos en la infancia. Sin embargo, se verifica que en Brasil existen pocos instrumentos que evalúen esta habilidad. Este estudio tiene como objetivo adaptar transculturalmente el Test de Conocimiento Emocional (EMT). El instrumento original fue traducido al portugués por un equipo de investigadores bilingues y, posteriormente, retraducido al inglés. Las versiones preliminares fueron juzgadas por los autores del instrumento y por jueces brasileros especialistas. La versión final fue aplicada a una muestra de 50 niños entre tres y seis años de edad y respondida por nueve jueces de tres estados brasileros. Los resultados muestran una buena equivalencia semántica, además de buenos niveles de concordancia con las respuestas $(\mathrm{k}=0,88 ; \mathrm{Z}=95,2 ; \mathrm{p}<0,001)$. La versión final del EMT se mostró adecuada y satisfactoria.

Palabras-clave: Emociones; Niños; Pruebas neuropsicológicas.
\end{abstract}

\section{Introdução}

Durante a infância, assim como no decorrer de todo o ciclo vital, as emoções desempenham um papel estruturante em todas as dimensões da atividade humana (Coll, Marchesi \& Palácios, 2004). Elas são essenciais para as interações sociais, sendo um dos recursos mais significativos que os bebês e crianças pequenas dispõem para comunicar os seus estados internos. É essencial que, desde o nascimento, os seres humanos compartilhem seus estados internos com parceiros sociais e sejam capazes de processar informações relativas ao estado afetivo dos mesmos (Bergamasco, 1997). Ainda nos primeiros meses de vida, o bebê começa a desenvolver a capacidade de percepção de emoções relativa às expressões vocais e faciais e, no decorrer da infância, 
as competências emocionais tornam-se gradativamente mais complexas (Bergamasco, 1997; Coll e cols., 2004; Flom, Gentile \& Pick, 2008; Izard, 2001; Morgan, Izard, \& King, 2009).

O conhecimento emocional (CE) é uma dimensão do desenvolvimento socioafetivo, que pode ser definido como a compreensão das "expressões, rótulos e funções das emoções" (Izard, 2001, p. 250). Sua aquisição favorece a estruturação de relações saudáveis do indivíduo com seu contexto, na medida em que auxilia na antecipação de sentimentos e comportamentos de outras pessoas e na expressão dos seus próprios sentimentos, aumentando "a probabilidade de que o indivíduo utilize a capacidade de adaptação inerente à excitação e à motivação emocional" (Izard, 2001, p. 250). Trata-se de um construto complexo, que possui múltiplas dimensões, entre as quais se encontram: a capacidade de perceber as emoções através de sinais em expressões faciais, vocais ou comportamentais em contextos diversos; rotulá-las e nomeá-las; identificar suas causas; conhecer as normas culturais para sua expressão; reconhecer dissimulação, ocorrências simultâneas e ambivalências; antecipar e reconhecer os seus próprios sentimentos ou compreender as relações entre emoção, motivação e comportamento (Izard, 2001).

A primeira infância, compreendida entre 0 a 6 anos de idade (UNICEF, 2008), é um "um período especialmente relevante para o desenvolvimento humano, em que habilidades cognitivas e psicossociais fundamentais são desenvolvidas" (Ferreira, Coutinho, Freitas, Malloy-Diniz \& Haase, 2010, p. 212). Aspectos do CE começam a se desenvolver desde os primeiros meses de vida, sendo que as diversas habilidades que o compõem emergem em diferentes etapas do desenvolvimento infantil. Em crianças pequenas, destaca-se: a capacidade de reconhecer expressões de emoções - CE receptivo; rotulá-las e nomeá-las - CE expressivo; e compreender as suas causas e consequências, associando eventos ambientais a rótulos ou tipos de emoção - Conhecimento de situações emocionais (Morgan e cols., 2009). Quanto ao tipo de emoção considera-se que, em termos ontogenéticos, habilidades referentes às emoções básicas - alegria, tristeza, raiva, medo, surpresa e nojo - são desenvolvidas desde os primeiros meses de vida, enquanto as emoções sociais - culpa, vergonha, orgulho, ciúme, entre outras - estão mais fortemente associadas a processos de aprendizagem e socialização (Ekman, 1992; Fernandéz-Abascal, Rodríguez, Sanchez, Díaz, \& Sánchez, 2010; Izard, 2009).
Atualmente, há um crescente interesse no estudo do CE e sua relação com outras dimensões do desenvolvimento humano, especialmente em crianças e adolescentes. Pesquisas recentes apontam que um maior CE relaciona-se positivamente com competência de regular as emoções (Morgan e cols., 2009; Trentacosta \& Izard, 2007), habilidades sociais (Schultz, Izard, Ackerman \& Youngstrom, 2001), controle inibitório (Morgan e cols., 2009) e desempenho escolar (Bennett, Bendersky, \& Lewis, 2005; Garner \& Waajid, 2008; Trentacosta \& Izard, 2007); o CE também correlaciona-se negativamente com níveis de agressividade (Denham e cols., 2002; Schultz, Izard \& Bear, 2004), interações negativas entre pares (Morgan e cols., 2009) e problemas de comportamento (Alonso-Alberca, Vergara, Fernandez-Berrocal, Johnson, \& Izard, 2012; Morgan e cols., 2009).

Dificuldades socioafetivas presentes na primeira infância, tais como introversão, agressividade, entre outras, tendem a se perseverar após este período. Déficit no processamento emocional tem sido associado a diversos transtornos neuropsiquiátricos (Denham,1998). O CE está prejudicado em crianças com autismo (Esposito, Nakazawa, Venuti \& Bornstein, 2012; Neves e cols., 2011; Philip e cols., 2010; Uljarevic \& Hamilton, 2012; Williams \& Gray, 2012); com transtorno bipolar pediátrico (Shankman. Katz, Passaroti \& Pavuluri, 2012) e com transtorno de ansiedade (Bender, Reinholdt-Dunne, Esbjørn \& Pons, 2012). O CE também mostra-se alterado em indivíduos com lesões e disfunções no lobo temporal, a exemplo da epilepsia do lobo temporal (Adolphs, Tranel, Damasio, \& Damasio, 1994, 1995; Batista \& Freitas Magalhães, 2009; Calder e cols., 1996; Phan, Wager, Taylor, \& Liberzo, 2001), na depressão (Bistricky, Ingram \& Atchley, 2012) e esquizofrenia (Kohler, Turner, Gur, \& Gur, 2004).

Em virtude da importância das emoções para os seres humanos, especialmente durante a infância, assim como a presença de deficit no processamento emocional em diversos transtornos neuropsiquiátricos, é necessário que existam instrumentos para sua avaliação no país. O Sistema de Avaliação de Testes Psicológicos (SATEPS, 2012) do Conselho Federal de Psicologia (CFP) conta com 130 testes psicológicos aprovados para uso. Entre eles, 40 têm como população-alvo as crianças. Entretanto, entre os testes aprovados pelo CFP, apenas 1,3\% tem como população-alvo crianças entre três de seis anos de idade e nenhum possui o objetivo de avaliar o desenvolvimento emocional em crianças até 6 anos de idade. 
A visível escassez de instrumentos para avaliação decrianças no Brasil é agravada por um contexto no qual muitos instrumentos utilizados são desenvolvidos em outros países e apenas traduzidos para o português (Argollo e cols., 2009; Ferreira e cols., 2010). Ao desconsiderar especificidades linguísticas e culturais, a tradução literal de testes pode revelar-se insuficiente. A adaptação de um instrumento portanto, exige um processo cuidadoso, que leve em consideração as especificidades linguísticas e semânticas do português no Brasil e contemple os diferentes contextos culturais e estilo de vida da população-alvo da versão. (Argollo e cols., 2009; Reichenheim \& Moraes, 2007). Ela deve abarcar as diferenças culturais tanto na adaptação dos itens quanto na interpretação de seus resultados. (Hambleton \& Patsula, 1998).

O processo de adaptação de testes deve considerar diversos aspectos, tais como a análise de equivalência conceitual e semântica, a convergência entre as versões originais e adaptadas, além da análise dos itens propriamente ditos(Malloy-Diniz e cols., 2010). Ao possibilitar uma contextualização para a cultura e população-alvo a qual se destina e, por sua vez, uma convergência com o instrumento original, a adaptação transcultural revela-se uma estratégia relevante para possibilitar o intercâmbio de informações entre a comunidade científica de diferentes países e a realização de pesquisas transculturais. (Argollo e cols., 2009; Hambleton \& Patsula, 1998).

O presente estudo descreve a primeira etapa de adaptação transcultural do Teste de Conhecimento Emocional (Emotion Matching Task - EMT). Desenvolvido por Izard, Hankins, Schultz, Tentracosta \& King (2003), este instrumento busca ser medida abrangente de CE, abordando os principais componentes desta função psicológica em crianças de 3 a 6 anos de idade. A escolha de adaptação deste teste ocorreu por diferentes motivos. Entre eles destacam-se: 1) avalia os componentes do CE mais relevantes para a faixa etária à qual se destina; 2) destina-se a crianças menores de seis anos de idade; 3) contêm fotografias de crianças contemplando diversidade étnica; 4) o conteúdo dos itens e os materiais de estímulos aparentam ser familiares à população-alvo; 5) pode ser utilizado para fins clínicos e de pesquisa.

\section{Materiais e métodos}

\section{Procedimentos para coleta de dados}

Este estudo foi composto de cinco fases (Figura 1) fundamentadas nas diretrizes propostas pela Resolução do CFP n 002/2003, bem como pelo guia da International Test Commission, - "ITC Guideline" (Vijver \& Hambleton, 1996). Na primeira fase, a versão original do EMT foi traduzida por três pesquisadores brasileiros bilíngues com experiência na atuação com crianças e formação em psicologia do desenvolvimento, neuropsicologia e neuropediatria. A opção por uma equipe para realizar a tradução está associada à orientação de que "a qualidade das traduções (...) pode beneficiar-se de uma abordagem em que não um único bilíngue, mas todo um grupo de pessoas participa do processo de tradução, particularmente quando este grupo combina a competência linguística e psicológica" (Vijver \& Hambleton, 1996, p. 94).

A segunda fase consistiu na tradução reversa do instrumento realizada por um professor de inglês de origem norte-americana fluente em português e inglês. A equivalência literal idiomática e semântica entre a retrotradução e a tradução, terceira fase, foi avaliada por

\section{Fase 1: Tradução e adaptação da versão original do EMT}

- Realizada por uma equipe com três profissionais bilíngues e especializados

\section{Fase 2: Tradução reversa do EMT}

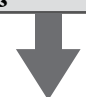

\section{Fase 3: Análise Semântica I}

- Análise da equivalência idiomática e semântica feita pelos autores do instrumento e três juízes brasileiros

\section{Fase 4: Análise semântica II}

- Estudo piloto (10 crianças de 3 anos e 10 crianças de 6 anos de idade)

Fase 5: Adaptação transcultural das respostas aos itens I

- Análise das respostas realizada por nove juízes da Bahia, Minas Gerais e São Paulo.

- Aplicação do EMT com 30 crianças de 6 anos de idade

Figura 1. Procedimentos para adaptação transcultural do EMT 
meio de uma escala likert de quatro pontos, que julga o conteúdo em 1 - "inalterado", 2 - "pouco alterado", 3 - "moderadamente alterado" e 4 - "muito alterado". Esta avaliação foi realizada pelos autores do instrumento, assim como por três juízes bilíngues, todos com titulação de doutorado.

A análise semântica compôs também a quarta fase deste estudo e teve por objetivo "verificar se todos os itens são compreensíveis para todos os membros da população à qual o instrumento se destina" (Pasquali, 1998, p. 210). Segundo Pasquali (1998), é relevante verificar se os itens são compreensíveis tanto pelos indivíduos com menor habilidade quanto pelos indivíduos com maior habilidade da população pretendida pelo instrumento. Portanto, a variante preliminar da versão brasileira do EMT foi aplicada em um estudo piloto com crianças das idades limítrofes, visando testar a compreensão das instruções e tarefas. Nesta ocasião, dois pesquisadores estiveram presentes com objetivo de realizar a entrevista e a observação dos comportamentos da criança.

Considerando tratar-se de um instrumento que avalia o CE, fez-se relevante a adaptação cultural das respostas. Para tal objetivo, na quinta fase, nove juízes com experiência em emoções, psicometria, neuropsicologia do desenvolvimento e psicologia clínica de três estados brasileiros, Bahia, Minas e São Paulo, responderam ao instrumento, sendo analisados os índices de concordância entre suas respostas. Quatro destes juízes possuem doutorado na área. Para o grupo com maior habilidade da população-alvo do EMT, foram selecionadas crianças de seis anos pertencentes a níveis socioeconômicos A1 e B1. Isso se deve à associação estabelecida por estudos anteriores entre condições socioeconômicas relacionadas à baixa renda familiar e menor habilidade em tarefas de CE (Schultz e cols., 2001).

Uma carta convite, juntamente com o termo de consentimento livre e esclarecido, foi encaminhada aos pais das crianças. Os mesmos responderam também a um questionário contendo informações sobre a saúde da criança e dados socioeconômicos da família. Os procedimentos adotados nesta pesquisa seguiram as orientações éticas previstas na Resolução 196/96, do Conselho Nacional de Saúde (1996), e na Resolução 016/2000, do Conselho Federal de Psicologia (CFP, 2000) e a mesma foi submetida à aprovação de Comitê de Ética em Pesquisa da Maternidade Climério de Oliveira / Universidade Federal da Bahia, sob protocolo número 057/2011.

\section{Participantes}

Participaram do presente estudo crianças de ambos os sexos, sem histórico de transtornos neuropsiquiátricos, estudantes de escolas públicas e privadas residentes no município de Salvador. Foram selecionadas duas instituições privadas e uma instituição pública por conveniência de proximidade e acessibilidade.

$\mathrm{Na}$ quarta fase, análise semântica, a amostra constituiu-se de 10 crianças de três anos e 10 crianças de seis anos de idade, sendo 50\% estudantes de escolas públicas e $50 \%$ estudantes de escolas privadas (Tabela 1). $\mathrm{Na}$ quinta fase, equivalência de itens, trinta crianças de seis anos de idade em sua maioria oriundos de famílias com nível socioeconômico entre as classes A1 e B1 $(93,4 \%)$ e estudantes de escolas privadas (Tabela 1).

\section{Instrumentos}

O Teste de Conhecimento Emocional (EMT) tem como público alvo crianças de 3 a 6 anos de idade. $\mathrm{O}$ EMT envolve a apresentação de fotografias coloridas de crianças de diversas etnias realizando expressões faciais das emoções básicas de tristeza, alegria, surpresa/raiva, medo, além de expressão facial neutra. Composto de quatro tarefas com 12 itens cada, este instrumento avalia três dos principais componentes do CE. As tarefas 1 (fazendo a correspondência entre duas expressões de uma mesma emoção)e 4 (fazendo a correspondência entre o rótulo verbal e uma das quatro expressões emocionais) avaliam o CE receptivo, enquanto a tarefa 2 (fazendo a correspondência entre a descrição verbal de um ativador emocional com uma das quatro expressões emocionais) afere o Conhecimento de situações emocionais e a tarefa 3 (rotulando expressões emocionais), o CE expressivo.

Salienta-se que as fotografias utilizadas no EMT foram padronizadas previamente em população americana e brasileira. No Brasil, imagens mostrando que $74,7 \%$ tiveram percentual concordância superior a $80 \%$, sendo $95,2 \%$ julgadas como expressando a mesma emoção na população brasileira e estadunidense (Andrade, Abreu, Duran, Veloso \& Moreira, 2013). O EMT foi validado anteriormente para a população estadunidense e espanhola, apresentando boa consistência interna, forte correlação com outras medidas que mensuram o mesmo construto e bons indicadores de validade de critério (Alonso-Albercae cols., 2012; Morgan e cols., 2009).

\section{Procedimentos para análise de dados}

As análises quantitativas foram realizadas utilizando os softwares SPSS (Statistical Package for Social 
Tabela 1. Características sociodemográficas dos participantes

\begin{tabular}{|c|c|c|c|}
\hline & \multicolumn{2}{|c|}{ Fase 4} & Fase 5 \\
\hline & \multicolumn{2}{|c|}{ Crianças } & \multirow{2}{*}{$\begin{array}{c}\text { Crianças com } 6 \text { anos } \\
N=30\end{array}$} \\
\hline & $\begin{array}{l}3 \text { anos } \\
N=10\end{array}$ & $\begin{array}{l}6 \text { anos } \\
N=10\end{array}$ & \\
\hline \multicolumn{4}{|l|}{ Sexo } \\
\hline Feminino & $50,0 \%$ & $40,0 \%$ & $43,3 \%$ \\
\hline Masculino & $50,0 \%$ & $60,0 \%$ & $56,7 \%$ \\
\hline Idade em meses & $M=42,70(D P=3,529)$ & $M=75,70(D P=3,802)$ & $M=77,00(D P=3,322)$ \\
\hline \multicolumn{4}{|l|}{ Classe econômica } \\
\hline A1 & $10,0 \%$ & $10,0 \%$ & $6,7 \%$ \\
\hline A2 & $0,0 \%$ & $10,0 \%$ & $50,0 \%$ \\
\hline B1 & $30,0 \%$ & $20,0 \%$ & $36,7 \%$ \\
\hline $\mathrm{B} 2$ & $0,0 \%$ & $20,0 \%$ & $6,7 \%$ \\
\hline $\mathrm{C} 1$ & $20,0 \%$ & $20,0 \%$ & $0,0 \%$ \\
\hline $\mathrm{C} 2$ & $30,0 \%$ & $20,0 \%$ & $0,0 \%$ \\
\hline $\mathrm{D}$ & $10,0 \%$ & $0,0 \%$ & $0,0 \%$ \\
\hline \multicolumn{4}{|l|}{ Tipo de escola } \\
\hline Pública & $50,0 \%$ & $50,0 \%$ & $3,3 \%$ \\
\hline Privada & $50,0 \%$ & $50,0 \%$ & $96,7 \%$ \\
\hline
\end{tabular}

Sciences), versão 20.0 e STATA (Data Analysis and Statistical Software), versão 10.0. Para analisar o acordo interjuízes foi utilizado o coeficiente Kappa para múltiplos juízes (Fleiss, 1971). O coeficiente Kappa de Cohen, assim como as suas variações, a serem utilizadas na presença de múltiplos juízes, é calculado para as análises de variáveis nominais, sendo amplamente utilizados no campo da psicologia (Fonseca, Silva \& Silva, 2007).

\section{Resultados}

\section{Equivalência conceitual e semântica}

O grupo de especialistas considerou as imagens das crianças pertinentes ao contexto brasileiro, visto que abrangia a maior parte das raças/etnias presentes no país: negra, indígena e branca. Avaliou-se que,em geral, os itens mostram-se adequados ao contexto sociocultural e ao cotidiano das crianças brasileiras.

A avaliação da tradução e retrotradução foi julgada pelos juízes como não alterada ou pouco alterada, média da escala likert=1,12 ( $\mathrm{DP}=0,22)$, indicando uma boa equivalência semântica do instrumento como todo. Optou-se pela conservação dos 48 itens originais. As instruções traduzidas para o português buscaram respeitar ao máximo a versão original do EMT, alterando ligeiramente as expressões linguísticas em escassas ocasiões, nas quais se avaliou como apropriado. Apenas o item 4 da tarefa 2 (versão original: "Show me the one who senice block tower was jus tkicked over by a meankid"), avaliado como uma situação pouco comum no contexto brasileiro, foi integralmente adaptado (versão adaptada: "Mostre a criança que acabou de ter seu grande castelo de areia destruído pelo seu colega malvado"). Como era esperado, este foi o item com maior média $(M=2,0, D P=0,8)$ na análise de juízes, sendo ainda considerado como pouco alterado. Em virtude da não adequação da tradução literal do título do instrumento, este foi adaptado com a colaboração dos autores.

A análise semântica, realizada por meio de entrevista individual com aplicação do instrumento em dez crianças de cada faixa etária limítrofe da população-alvo, indicou uma boa compreensão por parte da população-alvo tanto das instruções quanto dos itens. Não foram observados efeito piso ou teto nos participantes do grupo de menor ou maior habilidade, respectivamente. 


\section{Equivalência de itens}

Foi verificado o índice de concordância entre juízes e comparadas as respostas dos juízes brasileiros com as do instrumento original. Também foi analisada uma amostra de crianças com 6 anos de idade, parcela da população-alvo com maior habilidade no que se refere ao conhecimento emocional, tendo em vista a maturação dessa função no decorrer da primeira infância. As respostas dos juízes ao instrumento revelaram um excelente nível de concordância $(x=0,88, Z=95,2$, $p<0,001)$ e as crianças apresentaram um bom nível $(x=$ $0,67, \mathrm{Z}=199,11, p<0,001)$. A análise do índice de concordância por tarefa mostrou resultados semelhantes (Tabela 2).

$\mathrm{Na}$ tarefa $1,91,7 \%$ dois quesitos tiveram respostas semelhantes entre o instrumento original, os juízes e as crianças. Apenas o item de número 2 apresentou divergências na avaliação dos juízes: 44\% consideraram a resposta 4 como correta e $33 \%$ a resposta 3 . No caso das crianças de 6 anos de idade, $72,4 \%$ da amostra considerou a resposta 4 como a correta. Entretanto, a análise dos juízes está em consonância com os resultados obtidos no estudo padronização de imagens contidas no EMT para a população brasileira (Andrade e cols., 2013). A imagem estímulo foi avaliada por $62 \%$ da amostra brasileira como face de expressão neutra, entretanto $34,2 \%$ a avaliou como representam a emoção de medo/surpresa. A imagem da resposta 4, com $44 \%$ de juízes apontando-a como correta, foi avaliada por $78,8 \%$ dos brasileiros como uma face neutra (Andrade e cols., 2013). Já a resposta 3, apontada como a resposta correta por $33 \%$ dos juízes foi avaliada como representando medo/surpresa por $96,6 \%$ da população. Considerando esses dados, a versão brasileira do EMT apresenta duas possibilidades de resposta para este item, a imagem 4 e a imagem 3.

A tarefa 2 também teve $91,7 \%$ dos itens com respostas correspondentes entre o instrumento original, os juízes e as crianças. O item 1, "Mostre a criança que teve seu belo desenho rasgado por um colega malvado", obteve a resposta 1 por parte de 55,6\% dos juízes e $83,3 \%$ das crianças. Essa imagem foi padronizada como expressando raiva (Andrade e cols., 2013). Entretanto $44,4 \%$ dos juízes apontaram como resposta uma foto de uma criança triste (Andrade e cols., 2013), indicando que os juízes avaliaram que a situação descrita no item, na cultura brasileira, pode ter como consequência tanto emoções de raiva quanto de tristeza. As tarefas 3 e 4 obtiveram respostas coincidentes entre juízes, crianças e crivo original do Teste de Conhecimento Emocional, possuindo um excelente índice de concordância entre os juízes e um bom nível de concordância entre as crianças (Tabela 2).

\section{Discussão}

A tradução e adapatação de um instrumento deve ser sistemática e cuidadosa, contemplando mais do que a tradução por uma equipe de pesquisadores (Argollo e cols., 2009; Malloy-Diniz e cols., 2010; Reichenheim \& Moraes, 2007). Os resultados da análise da equivalência semântica, uma das etapas do processo de adaptação,

Tabela 2. Índice de concordância por tarefa do EMT

Índice de concordância

Tarefa

Juízes Crianças de 06 anos de idade

1. "Fazendo a correspondência entre duas expressões de uma mesma emoção"

$$
x=0,84(\mathrm{Z}=38,76, p<0,01) \quad x=0,70(\mathrm{Z}=107,6, p<0,001)
$$

2. "Fazendo a correspondência entre a descrição verbal de um ativador emocional com uma das quatro expressões

$$
x=0,73(\mathrm{Z}=26,46, \mathrm{p}<0,01) \quad x=0,53(\mathrm{Z}=65,5, \mathrm{p}<0,001)
$$
emocionais"

3. "Rotulando expressões emocionais"

$$
x=0,92(\mathrm{Z}=38,4, \mathrm{p}<0,01) \quad x=0,64(\mathrm{Z}=96,1, \mathrm{p}<0,001)
$$

4. "Fazendo a correspondência entre o rótulo verbal e uma das quatro expressões emocionais"

$$
x=0,95(\mathrm{Z}=32,3, \mathrm{p}<0,01) \quad x=0,71(\mathrm{Z}=82,9, \mathrm{p}<0,001)
$$


indicaram uma boa equivalência da versão original do EMT e da versão adaptada, além de uma boa compreensão por parte da sua população-alvo. Segundo Ximenes (2008), é necessário convergir a versão adaptada à realidade local ou regional e analisar o quanto se ganha ou se perde em termos de generalização e comparabilidade. Nesse sentido, a opção pela adaptação integral de um dos itens do instrumento levou em conta a realidade local. O mesmo foi avaliado pelos juízes como pouco alterado em relação à versão original, o que, possivelmente, indica pouca perda quanto à capacidade de comparabilidade. Esses resultados apontam que a versão traduzida e adaptada do EMT possui "capacidade de transferência de sentido dos conceitos contidos no instrumento original" (Reichenheim \& Moraes, 2007, p. 668).

Ao mesmo tempo, a análise conceitual realizada por um grupo de especialistas considerou os estímulos do EMT pertinentes ao contexto brasileiro e à população-alvo, tendo em vista que se trata de fotografias de crianças abarcam parte significativa das raças/etmias presentes no país. Entretanto, a ausência de indivíduos de origem asiática, presente de forma expressiva em alguns dos estados brasileiros, pode ser significativa, apontando a necessidade de estudos que avaliem a relação entre a etnia de origem da criança e o desempenho no EMT.

$\mathrm{O}$ instrumento avalia o CE através de fotografias de expressões faciais de emoções básicas. Apesar de caracterizadas por um padrão universal de mudanças no Sistema Nervoso Autônomo e Sistema Nervoso Central e, consequentemente, padrão de respostas fisiológicas distintas e sinais universais, diferenças culturais também são relatadas no que se refere à acurácia de reconhecimento e classificação de intensidade das expressões de emoções básicas (Andrade e cols., 2013; Ekman, 1992; Izard, 1991; Matsumoto, Hwang \& Yamada, 2010). Buscando contemplar esta dimensão, a equivalência de itens foi mensurada com base na análise de juízes e crianças brasileiras acerca das respostas adequadas a cada quesito do instrumento. Os resultados mostram um excelente nível de concordância entre os juízes e um bom nível de concordância entre as crianças. Esta diferença é esperada tendo em vista que, apesar de ser a parcela da população-alvo com maior habilidade, o conhecimento emocional ainda está em desenvolvimento e suas habilidades ainda estão se consolidando em crianças de seis anos de idade (Morgan e cols., 2009).

Entre os 48 itens do instrumento, apenas dois apresentaram baixo índice de concordância dos juízes diante do crivo de respostas original. Entretanto, em ambos a resposta com maior frequência coincidiu com a versão original. No que se refere à análise das tarefas como um todo, ainda com um bom nível, o menor índice de concordância foi encontrado na intitulada "Fazendo a correspondência entre a descrição verbal de um ativador emocional com uma das quatro expressões emocionais". Esta aborda o conhecimento acerca das causas e consequências das emoções, sendo, portanto o componente na qual a socialização em uma determinada cultura exerce maior influência.

A versão adaptada do Teste de Conhecimento Emocional - EMT para a língua portuguesa e cultura brasileira mostrou-se adequada. Entretanto, a amostra ainda reduzida e não aleatorizada de crianças participantes revela-se uma limitação do presente trabalho e não possibilita análise da validade deste instrumento. Nesse sentido, novas pesquisas são necessárias com vista a analisar as propriedades psicométricas do EMT, realizando uma análise acerca da sua validade de construto e critério, estrutura fatorial, capacidade de discriminar o desempenho de crianças em diferentes faixas etárias e análise do desempenho de um grupo normativo em diferentes estados brasileiros. Referidos estudos encontram-se em andamento e possibilitarão a utilização apropriada do EMT para fins clínicos e de pesquisa em contexto brasileiro.

\section{Referências}

Adolphs, R., Tranel, D., Damasio, H., \& Damasio, A. R. (1994). Impaired recognition of emotion in facial expressions following bilateral damage to the human amygdala. Nature, 372(6507), 669-672.

Adolphs, R., Tranel, D., Damasio, H., \& Damasio, A. R. (1995). Fear and the human amygdala. The Journal of Neurosciences, 15(9), 5879-5891.

Alonso-Alberca, N., Vergara, I., Fernandez-Berrocal, P., Johnson, S. R., \& Izard, C. E. (2012). The adaptation and validation of the Emotion Matching Task for preschool children in Spain. International Journal of Behavioral Development, 36(6), 489-494.

Andrade, N. C., Abreu, N., Duran, V. R., Veloso, T., \& Moreira, N. A. (2013). Reconhecimento de expressões faciais de emoções: padronização de imagens do teste de conhecimento emocional. Psico, 44(3), 382-390.

Argollo, N., Bueno, O. F. A., Shayer, B., Gondinho, K., Durán, P., Assis, A., .... Lima, F. (2009). Adaptação transcultural da Bateria NEPSY - avaliação neuropsicológica do desenvolvimento: estudo piloto. Avaliação Psicológica, 8(1), 59-75. 
Associação Brasileira de Empresas de Pesquisa (2011). Critério de classificação econômica Brasil. São Paulo.

Batista, J., \& Freitas-Margalhâes, A. (2009). The recognition of basic emotions in temporal lobe epilepsy. Em Freitas-Magalhães (2009). Emotional expression: the brain and the face. Porto: Edições Universidade Fernando Pessoa.

Bender, P. K., Reinholdt-Dunne, M. L., Esbjørn, B. H., \& Pons, F. (2012). Emotion dysregulation and anxiety in children and adolescents: gender differences. Personality and Individual Differences, 53(3), 284-288.

Bennett, D. S., Bendersky, M. \& Lewis, M. (2005). Antecedents of emotion knowledge: predictors of individual differences in young children. Cognitioneremotion, 19(3), 375-396.

Bergamasco, N. H. P. (1997). Expressão facial como acesso à consciência do recém-nascido. Psicologia USP, 8(2), 275-286.

Bistricky, S. L., Ingram, R. E., \& Atchley, R. Ann. (2011). Facial Affect Processing and Depression Susceptibility. Psychological Bulletin, 137(6), 998-1028.

Calder, A. J., Young, A. W., Rowland, D., Perrett, D. I., Hodges, J. R., \& Etcoff, N. L. (1996). Facial emotion recognition after bilateral amygdala damage: differentially severe impairment of fear. Neuropsychology, 13, 699-745.

Conselho Federal de Psicologia. (2000). Resolução CFP $n^{\circ}$ 016/2000 de 20 de dezembro de 2000. Dispõe sobre a realização de pesquisa em Psicologia com seres humanos. Brasília, DF.

Conselho Federal de Psicologia (2003). Resolução CFP $n^{\circ} 02 / 2003$ de 24 de marco de 2003. Define e regulamento o uso, a elaboração e a comercialização de testes psicológicos. Brasilia, DF.

Conselho Nacional da Saúde. (1996). Resolução no 196 de 10 de outubro de 1996. Diretrizes e normas regulamentadoras de pesquisas envolvendo seres humanos. Brasília, DF.

Coll, C., Marchesi, A., \& Palácios, J. (2004). Desenvolvimento psicológico e educaşão: psicologia evolutiva. Porto Alegre: Artmed.

Denham, S. A. (1986). Social cognition, prosocial behavior, and emotion in preschoolers: contextual validation. Child Development, 56, 197-201.

Denham, S. A. (1998). Emotional development in young children. Nova Iorque Londres: The Guilford Press.
Denham, S. A, Caverly, S., Schmidt, M., Blair, K., DeMulder, E., Caal, S., ... Hamada, H.(2002). Preschool understanding of emotions: contributions to classroom anger and aggression. Journal of Child Psychology and Psychiatry, and Allied Disciplines, 43(7), 901-16.

Ekman, P. (1992). An argument for basic emotions. Cognition and emotion, 6(3-4), 169-200.

Esposito, G., Nakazawa, J., Venuti, P., \& Bornstein, M. H. (2012). Perceptions of distress in young children with autism compared to typically developing children: a cultural comparison between Japan and Italy. Research in Developmental Disabilities, 33(4), 1059-1067.

Fernandéz-Abascal, E. G., Rodríguez, B. G., Sanchez, M. P. J., Díaz, M. D. M., \& Sánchez, F. J. D. (2010). Psicologia de la emoción. Madrid: Editorial Ramón Areces.

Ferreira, F. O., Coutinho, G., Freitas, P. M., MalloyDiniz, L. F., \& Haase, V. G. (2010). O exame neuropsicológico na idade pré-escolar. Em N. Malloy-Diniz, L. F., Fuentes, D., Mattos, P., Abreu (Ed.), Avaliação Neuropsicológica (pp. 210-220). Porto Alegre: Artmed.

Fleiss, J. L. (1971). Measuring nominal scale agreement among many raters. Psychological Bulletin, 76(5), 378-382.

Flom, R., Gentile, D. A, \& Pick, A. D. (2008). Infants' discrimination of happy and sad music. Infantbehavior \& Development, 31(4), 716-28.

Fonseca, R., Silva, P., \& Silva, R. (2007). Acordo interjuízes : o caso do coeficiente Kappa. Laboratório de Psicologia, 5(1), 81-90.

Fonseca, D., Seguier, V., Santos, A., Poinso, F., \& Deruelle, C. (2009). Emotion understanding in children with ADHD. Child psychiatry and buman development, 40(1), 111-21.

Garner, P., \& Waajid, B. (2008). The associations of emotion knowledge and teacher-child relationships to preschool children's school-related developmental competence. Journal of Applied Developmental Psychology, 29(2), 89-100.

Hambleton, R. K., \& Patsula, L. (1998). Adapting tests for use in multiple languages and cultures. Social Indicators Research, 45(1-3), 153-171.

Izard, C. E. (1991). The psychology of emotions. Nova Iorque: Plenum 
Izard, C. E. (2001). Emotional intelligence or adaptive emotions? Emotion, 1(3), 249-257.

Izard, C. E. (2009). Emotion Theory and Research: highlights, unanswered questions and emerging issues. Annu Rev Psychol., 60, 1-25.

Izard, C. E., Haskins, F. W., Schultz, D., Trentacosta, C. J., \& King, K. A. (2003). Emotion Matching Task. Unpublished test (Contains 182 standardized color photos of children's emotion expressions). Newark, DE: University of Delaware.

Kohler, C. G., Turner, T. H., Gur, R. E., \& Gur, R. C. (2004). Recognition of facial emotions in neuropsychiatric disorders. CNS spectrums, 9(4), 267-74.

Malloy-Diniz, L. F., Mattos, P., Leite, W. B., Abreu, N., Paula, J. J. De, Tavares, H., ... Fuentes, D. (2010). Tradução e adaptação cultural da Barratt Impulsiveness Scale (BIS-11) para aplicação em adultos brasileiros. J BrasPsiquiatria, 59(2), 99-105.

Matsumoto, D., Hwang, H. S., \& Yamada, H. (2010). Cultural differences in the relative contributions of face and context to judgments of emotions. Journal of Cross-Cultural Psychology, 43(2), 198-218.

Morgan, J. K., Izard, C. E., \& King, K. A. (2009). Construct Validity of the Emotion Matching Task: preliminary evidence for convergent and criterion validity of a new emotion knowledge measure for young children. Social development (Oxford, England), 19(1), 52-70.

Neves, M. de C. L.,Tremeau, F., Nicolato, R., Lauar, H., Romano-Silva, M. A., \& Correa, H. (2011). Facial emotion recognition deficits in relatives of children with autism are not associated with 5HTTLPR. Rev. Bras. Psiquiatr., 33(3), 261-267.

Pasquali, L. (1998). Princípios de elaboração de escalas psicológicas. Revista de Psiquiatria Clínica, 25(5), 206-213.

Phan, K. L., Wager, T., Taylor, S. F., \& Liberzo, L. (2002). Functional Neuroanatomy of Emotion: a meta-analysis of emotion activation studies in pet and fmri1, Neuroimage, 16(2), 331-348.

Philip, R. C., Whalley, A. C., Stanfield, R., Sprengelmeyer, I. M. Santos, A. W. Young, A. P. ... Atkinson. (2010). Déficits in facial, body movement and vocal emotional processing in autism spectrum disorders. Psychological Medicine, 40, 19191929.
Reichenheim, M. E., \& Moraes, C. L. (2007). Operacionalização de adaptação transcultural de instrumentos de aferição usados em epidemiologia. Cadernos de Saúde Pública, 41(4), 665-673.

SATEPSI - Sistema de Avaliação de Testes Psicológicos (2012). Lista Completa de testes. Disponível em http://www2.pol.org.br/satepsi/sistema/admin.cfm?lista $2=\operatorname{sim}$

Schultz, D., Izard, C. E., Ackerman, B. P., \& Youngstrom, E. A. (2001). Emotion knowledge in economically disadvantaged children: self-regulatory antecedents and relations to social difficulties and withdrawal. Development and Psychopathology, 13, 53-67.

Schultz, D., Izard, C. \& Bear, G. (2004) Children's emotion processes: relations to emotionality and aggression. Development and Psychopatology, 16(2), 371-387.

Shankman, S. A., Katz, A. C., Passarotti, A. M., \& Pavuluri, M. N. (2012). Déficits in emotion recognition in pediatric bipolar disorder: The mediating effects of irritability. Journal of Affective Disorders, 140(1). 165-327.

Trentacosta, C. J., \& Izard, C. E. (2007). Kindergarten children's emotion competence as a predictor of their academic competence in first grade. Emotion (Washington, D.C.), 7(1), 77-88.

UNICEF - Fundo das Nações Unidas para a Infância (2008). A transição dos cuidados na primeira infância, Innocenti Report Card, no 8 . Florença: Centro de Pesquisa Innocenti UNICEF.

Uljarevic, M. \& Hamilton, A. (2012). Recognition of emotions in autism: a formal meta-analysis. J. Autism Dev Disord, 43(7), 1517-1526.

Vijver, F. V. D., \& Hambleton, R. K. (1996).Translating tests : some practical guidelines. Options, 1(2), 89-99.

Williams, B. T. \& Gray, K. M. (2012).The relationship between emotion recognition ability and social skills in young children with autism. Autism. O(0), 1-7.

Ximenes, L. B. (2008). Adaptação transcultural para o Brasil da Exercise Benefits / Barriers Scale (EBBS) para aplicação em idosos: uma avaliação semântica. Aging, 24(12), 2852-2860.

Recebido em: 05/04/2013

Reformulado em: 10/01/2014

Segunda reformulação em: 31/03/2014

Aprovado em: 10/04/2014 
Nota dos autores:

Agradecimentos: Agrademos ao Professor Dr. Carroll Izard e a sua equipe, por disponibilizarem o instrumento e construírem conjuntamente este trabalho. Aos Professores Nayara Argollo, Sônia Gondim, Marcos Emanoel Pereira e Leandro Malloy-Diniz, pelas constantes colaborações. Ao Professor Vitor Geraldi Haase e aos psicólogos Lorenzo Natale, Luiza Scaramuzza, Adriana Rossi e Silvia Feldberg, pela avaliação da retrotradução do EMT. À equipe do Laboratório de Neuropsicologia Clinica e Cognitiva, pela colaboração com a coleta de dados.

Sobre os autores:

Nara Côrtes Andrade é psicóloga, especialista em Social Therapeutic Methodology in Clinical and Comunitary psychology pelo East Side Institute for Group and Short Time Term Psychoteraphy, mestre em Psicologia do Desenvolvimento pela Universidade Federal da Bahia (2013), doutoranda em Psicologia Experimental pela Universidade de São Paulo, além de professora da Universidade Católica do Salvador.

Neander Abreu é psicólogo, especialista em Neuripsicologia, doutor em Neurociências e Comportamento, professor adjunto do Instituto de Psicologia da Universidade Federal da Bahia e coordenador do Laboratório de Neuropsicologia Clínica e Cognitiva - Neuroclic

Igor Menezes é professor adjunto do Instituto de Psicologia da Universidade Federal da Bahia (UFBa) na área de Psicometria e do Programa de Pós-Graduação em Psicologia (PPGPSI/UFBa), bolsista de Produtividade em Pesquisa do CNPq, coordenador do Núcleo de Instrumentos e Medidas (NIM/UFBa). Coordena pesquisas sobre construção e validação de medidas psicológicas, com ênfase no emprego da Teoria de Resposta ao Item.

Claudia Berlim de Mello é psicologa, especialista em Neuropsicologia, mestre em Psicologia (Desenvolvimento humano no contexto sociocultural) pela UnB, doutora em Psicologia (Neurociências e Comportamento) pela USP, coordenadora dos ambulatórios de Neuroplasticidade e Genética do Comportamento do Núcleo de Atendimento Neuropsicológico Infantil-NANI, do Centro Paulista de Neuropsicologia (Associação Fundo de Incentivo à Pesquisa -AFIP), e professora orientadora da Pós Graduação em Educação e Saúde, Campus Guarulhos-UNIFESP.

Victor Riccio Duran é graduando em Psicologia pela Universidade Federal da Bahia e participou de três projetos PIBIC, dois como voluntário e um como bolsista pela FAPESB (2010, 2011 e 2012). Bolsista de Graduação Sanduíche pelo Programa Ciência sem Fronteiras na University of Victoria, no Canadá (2013), foi academic visitor no Psychometrics Centre, University of Cambridge, no Reino Unido (janeiro a março, 2014).

Narena de Alencar Moreira é graduanda em Psicologia na Universidade Federal da Bahia, bolsista pelo Ciência sem Fronteiras na Michigan State University.

Contato com os autores:

Nara Andrade

Av. Ademar de Barros, s/n - Pavilhão 04

Campus Universitário de Ondina

CEP 40170-110, Salvador, BA, Brasil

Fone: (+5571) 3283-6474-8834-2438

E-mail: nandrade@usp.br 